\title{
Une action possible des sols sur la prédisposition des pêchers au dépérissement bactérien par modification de la teneur en eau hivernale des tiges
}

\author{
A Vigouroux ${ }^{1 *}$, C Bussi ${ }^{2}$ \\ 1 INRA, laboratoire de biologie et pathologie végétales, 2, place Viala, F34060 Montpellier cedex 1; \\ 2 INRA-SRIV, domaine expérimental de Gotheron, F26320 Saint-Marcel-lès-Valence, France
}

(Reçu le 29 juillet 1993; accepté le 6 mai 1994)

\begin{abstract}
Résumé - Nous avions montré antérieurement que l'infiltration hydrique des tissus corticaux de pêcher, qui survient à la fin des périodes de gel, permettait le développement d'infections bactériennes hivernales dues à Pseudomonas syringae pv persicae. Nous avons ensuite établi que ce phénomène et les infections associées sont plus importants lorsque la teneur en eau des tissus est plus élevée. Dans cette étude, nous avons pu modifier la sensibilité de pêchers cultivés en conteneur en modifiant légèrement leur teneur en eau uniquement en hiver (coefficient de corrélation : teneur en eau-lésion : $r=+0,698$ ). En parallèle, nous avons constaté que la teneur en eau hivernale de rameaux de pêcher cultivés sur des sols à texture grossière était supérieure, de l'ordre de $1,5 \%$, à celle des rameaux de pêchers cultivés sur sols fins. Cette bactériose est par ailleurs connue pour n'être grave que sur les sols grossiers. Il est conclu que la nature des sols pourrait influencer la sensibilité des pêchers à la bactériose, entre autres en modifiant la teneur en eau des arbres en hiver. Des résultats analogues seraient à attendre pour les autres bactéries hivernales des arbres fruitiers à noyau qui, comme d'ailleurs beaucoup d'autres ligneux, sont tous affectés par l'infiltration hydrique des tissus après gel et la diffusion de germes qui lui est associée.
\end{abstract}

gel / Prunus persica / Pseudomonas syringae / potentiel hydrique du sol / racine

Summary - Possible influence of coarse soils on the predisposition of peaches to bacterial die-back by increasing the water content of stem tissues in winter. We have previously indicated that frost-related water-soaking of peach cortical tissues induced winter Pseudomonas infection. We also established that this phenomenon was more important when the stem water content was higher. In this study, the winter water content and the susceptibility of the plants changed according to whether containers of one-year-old peach trees were covered in winter (correlation coefficient $r=+0.698$ ). In parallel, we found that the winter water content of peach twigs was higher (about $1.5 \%$ ) when trees were growing on coarse-structured soils favorable to the disease, compared with twigs of trees on fine-structured soils. These elements suggest that physical aspects of soils can influence tree susceptibility by altering their winter water content. Similar results are to be expected for other winter bacterial die-backs of stone-fruit trees because they are affected by frost-related water soaking and associated germ diffusion.

frost / Prunus persica / Pseudomonas syringae / environmental conditions / root system

* Correspondance et tirés à part 


\section{INTRODUCTION}

Nous avons antérieurement constaté que le dépérissement bactérien du pêcher provoqué par la bactérie Pseudomonas syringae pv persicae (Luisetti et al) Young et al était beaucoup plus grave dans les vergers installés sur sols acides à texture grossière (Vigouroux et Huguet, 1980). À l'étranger, des observations impliquant un rôle du sol dans le développement de maladies voisines ont aussi été rapportées (Davis et English, 1969 ; Weaver et Wehunt, 1975). L'étude entreprise pour expliquer le phénomène nous a permis dans un premier temps de montrer que la forte proportion d'éléments grossiers du sol constitue bien un facteur de prédisposition des arbres en induisant un stress hydrique estival et une perturbation de la nutrition calcique. Une corrélation positive a été trouvée entre taux de calcium et résistance des pêchers (Vigouroux et al, 1987 ; Vigouroux et Bussi, 1989). Ces résultats n'expliquaient cependant qu'une partie des phénomènes observés et des études parallèles nous ont conduits à envisager un autre mode d'action favorisante des sols grossiers qui se situerait, lui, en hiver et ferait appel à des mécanismes différents.

L'ensemble des bactérioses à Pseudomonas affectant les arbres fruitiers à noyaux, dont fait partie le dépérissement bactérien du pêcher, voit les infections se développer durant l'hiver et l'action du froid pour favoriser ce développement a été plusieurs fois soulignée (Klément et al, 1974 ; Vigouroux, 1974, 1979 ; Weaver, 1978). L'action de lésions dues au gel a été évoquée. En fait cela n'est justifiée qu'à propos d'infections printanières sur organe herbacé et pour des maladies proches (Panagopoulos et Crosse, 1964) avec probablement intervention de l'effet glaçogène des bactéries impliquées (Maki et al, 1974; Luisetti et Gaignard, 1985; Gaignard et Luisetti, 1993). Mais, en plein hiver, les mécanismes sont différents car les tissus des arbres, acclimatés comme le sont normalement nos arbres fruitiers, ne sont pas lésés par le gel. Pour Klément et al (1984), le développement des bactéries provoque une diminution de la teneur en sucres des tissus, ce qui alors les rendrait sensibles au gel (Levitt, 1956 ; Sakai et Yoshida, 1968). L'effet glaçogène des bactéries a aussi été invoqué à ce stade (Weaver et al, 1981 ; Luisetti et Gaignard, 1985) mais non confirmé. Personnellement, nous avons constaté qu'à la fin du gel l'état d'infiltration hydrique des tissus corti- caux, réalisé par la fonte de la glace accumulée dans les espaces intercellulaires (Geneves, 1955 ; Olien, 1967; Burke et al, 1976), peut entraîner une diffusion importante des germes à ce niveau (Vigouroux, 1989). Un développement rapide des infections s'ensuit si les températures continuent de monter quelque peu (Vigouroux, 1991a).

Plus récemment, nous avons montré que cette diffusion des germes est plus étendue et les infections bactériennes nettement plus graves lorsque la teneur en eau des tissus des arbres est plus élevée (Vigouroux, 1991b et 1994, article soumis). En outre, Young en 1974 a montré que la multiplication bactérienne dans les méats intercellulaires des tissus infectés est plus importante lorsque ces méats contiennent de l'eau. Or, diverses observations nous ont suggéré que des pêchers poussant sur des sols à texture grossière avaient, en hiver, des tissus plus riches en eau que ceux poussant sur sols à éléments fins ; ce qui pourrait alors constituer un autre mode de prédisposition des pêchers à la bactériose. Pour vérifier cette hypothèse, nous avons d'abord, avec des pêchers cultivés en conteneur, cherché à voir si une variation hivernale de leur teneur en eau par le biais d'une modification uniquement hivernale des propriétés du substrat de culture entraînait une variation de la sensibilité des arbres. Ensuite, au verger, nous avons mesuré tout au cours de l'hiver la teneur en eau de pêchers poussant sur sols fins et grossiers afin de vérifier que cette teneur était différente selon les sols.

\section{MATÉRIELS ET MÉTHODES}

L'étude a porté sur le pêcher, Prunus persica (L) Batsch, et, pour les observations de terrain, a été menée dans la moyenne vallée du Rhône. Cette zone est climatiquement favorable au dépérissement bactérien et la juxtaposition des sols d'origine diverses qu'on y rencontre (Bornand, 1968) permet la comparaison des influences édaphiques sous des conditions climatiques très similaires.

\section{Étude avec des arbres cultivés en conteneur}

Des scions du cv Golo, greffés sur porte-greffe GF 305 , ont été cultivés un an de façon séparée dans des conteneurs de $300 \mathrm{I}$ remplis d'une terre caillouteuse (cf analyse granulométrique au tableau I) prédisposant les arbres à la maladie (Vigouroux et Huguet, 1980). Un 
Tableau I. Granulométrie de la terre caillouteuse utilisée pour la culture des pêchers en conteneur.

\begin{tabular}{lll}
\hline & $\%$ & \%० \\
\hline Graviers et cailloux & 45 & \\
Fraction fine $(<2 \mathrm{~mm})$ & 55 & \\
$\quad$ Argile & & 191 \\
Limons fins & & 179 \\
Limons grossiers & & 141 \\
Sables fins & & 224 \\
Sables grossiers & 265 \\
\hline
\end{tabular}

lot de 24 arbres, inclus dans un dispositif plus vaste et disposé sur 2 rangs parallèles, a reçu les mêmes soins culturaux durant la saison de végétation (irrigation localisée, fertilisation, traitements phytosanitaires...). Pour différencier la teneur en eau hivernale, en nous inspirant d'un essai antérieur (résultats non publiés), nous avons, en automne au moment de la chute des feuilles, recouvert le dessus des bacs de 12 de ces arbres à l'aide d'un film de polyéthylène resserré autour du pied des troncs afin de protéger la terre des bacs de la pluie. Bacs protégés ou découverts étaient alternés sur le rang et placés en vis-à-vis d'un rang à l'autre. Juste après cette opération de recouvrement, nous avons inoculé chaque arbre par piqûre sur 5 rameaux identiques d'un an avec une aiguille lancéolée trempée dans une suspension de bactéries $\left(10^{6}\right.$ germes $/ \mathrm{ml}$ ) obtenue à partir d'une culture de la souche S32 de Ps s pv persicae $24 \mathrm{~h}$ sur milieu LPGA (Ridé, 1963). À la fin de février, nous avons estimé la teneur en eau des arbres en prélevant 12-13 rameaux répartis sur 2 arbres de même sorte et placés vis-à-vis entre chaque rang (la récolte sur un seul arbre de petite taille nous paraissait insuffisante pour obtenir un résultat valable). La teneur en eau a été déterminée en comparant poids frais et poids sec après passage au four à $80^{\circ} \mathrm{C} 48 \mathrm{~h}$. Quelques jours plus tard, la longueur des lésions bactériennes initiées par les piqûres et développées au cours de l'hiver a été mesurée. Compte tenu du faible développement des lésions cette année-là, la mesure a été faite après avoir pelé l'épiderme pour accéder à la nécrose des tissus sousjacents et avoir des mesures plus précises. On a regroupé et lait la moyenne des mesures des 10 inoculations portées par les couples d'arbres constitués juste avant pour estimer la teneur en eau. On a ainsi obtenu 6 valeurs par traitement et les moyennes de ces valeurs pour les 2 traitements ont été comparées statistiquement par le test $\varphi$ de Student. On a comparé de même les moyennes des teneurs en eau.

\section{Étude au verger sur sols différents}

Les mesures ont été faites avec comme support 2 cultivars de pêcher, Springlady et $O$ Henry, greffés sur $G$ $F 305$, les arbres étant âgés respectivement de 7 et 8 ans. Tous 2 étaient cultivés d'une part sur sols à éléments fins, d'autre part sur sol grossier, les parcelies correspondant à chaque type de sol étant distantes de quelques kilomètres. Les sols à éléments fins étaient constitués de limons d'alluvions récentes du Rhône ne contenant pratiquement aucun caillou limons fréquemment utilisés comme supports de vergers de pêchers dans cette région. Le système racinaire des arbres concernait une épaisseur de sol d'environ $1 \mathrm{~m}$, pratiquement homogène sur le plan textural. Les sols grossiers ont été formés sur alluvions anciennes fluvio-glaciaires très caillouteuses (Bornand, 1978), elles aussi cultivées en pêcher tout le long de la vallée du Rhône (Gras, 1962). Le système racinaire était presque complètement localisé au premièr horizon à fraction fine limono-sableuse, d'envrion $60 \mathrm{~cm}$ d'épaisseur et correspondant d'ailleurs à peu près à la couche de sol défoncée avant plantation. L'horizon sous-jacent bien qu'un peu plus argileux au niveau de la terre fine était tout aussi caillouteux et correctement drainant. L'analyse granulométrique de la fraction tine des sols des 4 parcelles a été portée dans le tableau II. Les différences portent surtout sur les sables grossiers mais on doit ajouter, pour les 2 sols "grossiers", les cailloux et graviers

Tableau II. Granulométrie des terres fines et grossières des parcelles supportant l'étude de la teneur en eau hivernale de 2 cultivars de pêcher.

\begin{tabular}{|c|c|c|c|c|}
\hline \multirow{2}{*}{$\begin{array}{l}\text { Dimensions } \\
\text { des particules }\end{array}$} & \multicolumn{2}{|c|}{ Spring Lady } & \multicolumn{2}{|c|}{ OHenry } \\
\hline & Sol fin * & Sol grossier ** & Sol fin * & Sol grossier ** \\
\hline$<2 \mu$ & 12 & 8 & 14 & 10 \\
\hline 2 à 20 & 22 & 19 & 21 & 17 \\
\hline 20 à 50 & 20 & 16 & 18 & 19 \\
\hline 50 à 200 & 44 & 22 & 39 & 23 \\
\hline 200 à 2000 & 2 & 35 & 8 & 31 \\
\hline
\end{tabular}

\footnotetext{
* Toute la terre a une granulométrie pratiquement $<2 \mathrm{~mm}$; ${ }^{* \star}$ la fraction fine $<2 \mathrm{~mm}$ ne représente que $50 \%$ du poids de la terre.
} 
constituant 45 à $50 \%$ du volume total de la terre. Les 4 parcelles appartenaient au même propriétaire et ont subi les mêmes techniques culturales. Malgré cela, les parcelles sur sol fin n'ont jamais été affectées par le dépérissement bactérien du pêcher alors que celle sur sol grossier ont antérieurement connu plusieurs épisodes de maladie.

On a évalué la teneur en eau des arbres par des prélèvements de rameaux échelonnés sur les mois de décembre à février pour Springlady et de janvier à février pour O Henry. Chaque prélèvement consistait, pour un cultivar sur un type de sol, en 4 lots de 15 rameaux récoltés à raison de 2 à 4 rameaux par arbre, à mi-hauteur et sur tout le pourtour de la couronne. On choisissait des rameaux "mixtes" de 40 à $45 \mathrm{~cm}$ de long, portant une proportion de bourgeons à fleur sensiblement identique pour chaque cultivar entre chaque type de sol. Sitôt récoltés, les rameaux étaient enfermés en sac plastique et ramenés en laboratoire. Ils y étaient immédiatement pesés puis déshydratés par un séjour de $48 \mathrm{~h}$ dans un four réglé à $80^{\circ} \mathrm{C}$ et repesés pour obtenir le poids sec. La teneur en eau initiale en était déduite en faisant la moyenne des 4 prélèvements qui permettaient un calcul de la ppds.

\section{RÉSULTATS}

\section{Étude en conteneur}

Le tableau III expose les moyennes des teneurs en eau et des longueurs de lésions relevées chez les arbres avec conteneur à ouverture libre (témoin) ou couvert. Les teneurs en eau et les lésions sont significativement $(P<0,025)$ plus grandes dans le cas des arbres témoins.

Si on considère les valeurs de ces éléments pour chacun des 12 couples d'arbres à conteneur couvert ou découvert, le coefficient de corrélation entre teneur en eau et longueur de lésion est $r=+0,698$.

\section{Étude au verger sur sols différents}

L'évolution comparée des teneurs en eau des rameaux des arbres sur les 2 types de sol et pour les 2 cultivars est exposée dans les graphiques de la figure 1.

On y voit que tout au cours de l'hiver (sauf pour le premier prélèvement sur Springlady) la teneur en eau est très significativement supérieure pour les rameaux des arbres installés sur sol grossier, et ce pour les 2 cultivars.

\section{DISCUSSION ET CONCLUSION}

\section{Arbres en conteneur}

Les inoculations réalisées sur les arbres en conteneur ont entraîné des lésions d'une taille très limitée, certainement en relation avec le manque de froid qui a caractérisé l'hiver de l'expérimentation. Cependant, les différences entre traitements sont nettement significatives $(P<0,025)$. Les tailles des lésions et les écarts ont d'ailleurs été beaucoup plus importants lors d'un essai antérieur identique (mais les teneurs en eau des arbres n'avaient été que sommairement estimées ; résultats non publiés). La différence entre les niveaux de teneur en eau est très faible mais elle aussi nettement significative $(P<$ $0,025)$. Il semble qu'elle puisse être attribuée à la différence entre les tensions de l'eau dans la terre des conteneurs, réhumectée ou non par la pluie. Trois tensiomètres répartis dans 3 conteneurs par traitement indiquaient à la fin de l'essai des valeurs comprises entre 12 et $16 \mathrm{cb}$ pour les conteneurs découverts et 32 à $37 \mathrm{cb}$ pour les bacs couverts. Un tel écart de tension de l'eau

Tableau III. Comparaison des teneurs en eau hivernales et des longueurs de lésions bactériennes obtenues chez des pêchers cultivés en conteneur couvert ou non en hiver.

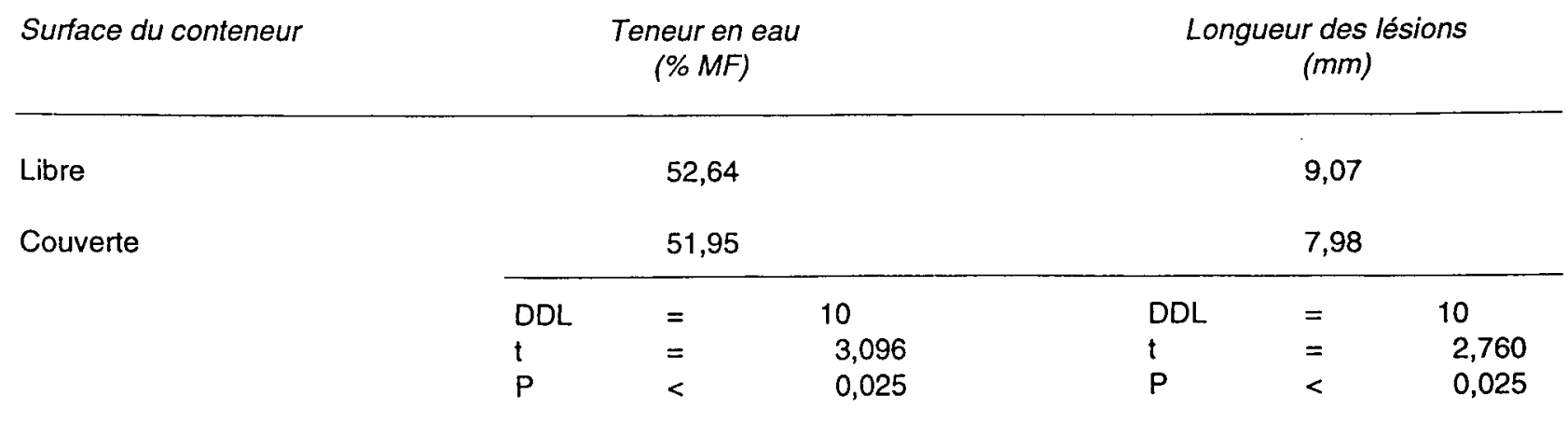




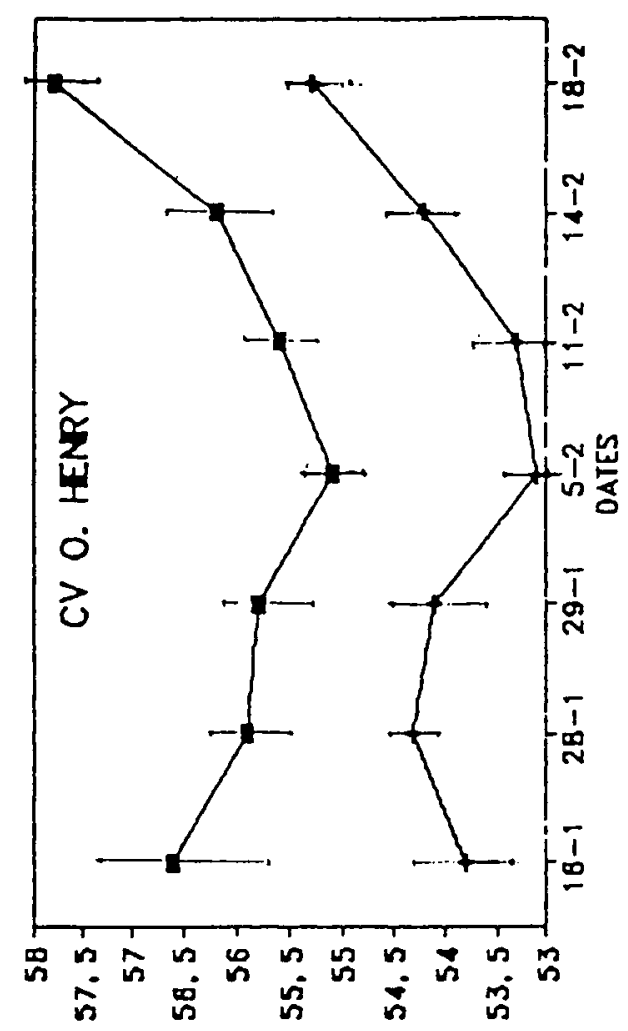


pourrait être suffisant, compte tenu de la faible activité racinaire en hiver (voir plus loin) pour limiter l'alimentation hydrique des arbres en conteneur couvert.

Quoi qu'il en soit, il apparaît qu'une intervention uniquement au niveau du substrat et seulement durant l'hiver a réduit la teneur en eau des arbres et en même temps entraîné une réduction des infections bactériennes sur rameaux. Le niveau très élevé de la corrélation positive, entre les valeurs de teneur en eau et les longueurs de lésion, semble confirmer l'association des 2 éléments.

\section{Teneur en eau selon les sols des vergers}

La teneur en eau des rameaux des arbres est assez stable (Wiegand, 1906). L'écart entre rameaux de pêchers cultivés sur sols fins et grossiers qui se maintient entre 1 et $2 \%$ tout au cours de l'hiver est remarquable. L'évolution des 2 teneurs reste parallèle, même lors de variations ponctuelles ou plus prolongées dans le temps, simultanées pour les 2 types d'arbres (ces variations, très importantes, ont pu être associées à des événements climatiques, gels, périodes froides, réchauffement printanier...). L'écart non significatif pour le premier prélèvement de Spring Lady est dû à un mauvais échantillonnage, amélioré par la suite.

Des récoltes et mesures ponctuelles réalisées avec d'autres cultivars (Aline, Springcrest), sur des arbres d'âges variés et dans une zone de culture un peu plus septentrionale, ont fourni entre sols fins et sols grossiers des écarts de teneur en eau du même ordre et dans le même sens. On a déjà souligné par ailleurs combien les sols des parcelles étudiées étaient représentatifs des 2 types de sols portant des pêchers dans toute la vallée du Rhône. On peut donc penser que ces variations de teneur en eau constituent un phénomène général associé au mode de fonctionnement racinaire lié aux caractéristiques édaphiques des sols, donc de leurs structures.

De façon générale, en comparaison de l'été, I'hiver est caractérisé par des conditions humides avec a priori une eau abondante au niveau du sol et une faible demande aérienne. Mais cette dernière est réelle (elle provoque par exemple un début de dessèchement en $24 \mathrm{~h}$ chez un rameau coupé et laissé à l'air) alors que les températures basses de l'hiver diminuent fortement l'activité racinaire. Or, dans un contexte d'eau abondante, les caractéristiques granulométriques entraînent aussi des différences importantes entre les sols fins et grossiers. Dans les sols à éléments fins, la microporosité est le plus souvent totalement occupée par l'eau, et la macroporosité qui, seule, reste remplie d'air après drainage est, par constitution, extrêmement limitée.

L'oxygénation est donc faible. En outre, le diamètre réduit de la porosité freine la circulation de l'eau, diminuant encore les possibilités d'oxygénation. Cependant, les arbres ne souffrent pas vraiment de cette anoxie, se trouvant en vie ralentie. Dans les sols grossiers, la macroporosité est importante et l'oxygénation bien supérieure (Lévy et Toutain, 1979), les écarts étant encore plus marqués lorsqu'il s'agit de matériaux peu argileux (Chrétien et Tessier, 1988), ce qui est le cas pour les sols des vergers étudiés. D'autre part, les éléments fins ont un pouvoir de succion de l'eau plus élevé que celui des particules grossières, sauf en périodes transitoires de saturation. La tension de l'eau dans les sols fins est donc, a priori, plus forte que celle de l'eau des sols grossiers, même lorsqu'on s'approche de la capacité au champ (Marty, 1969 ; Yong et Warkentin, 1975 ; Fitter et Hay, 1987). Ce phénomène constitue un frein à l'absorption racinaire, de même que le manque d'oxygène évoqué juste avant, ce qui réduit l'activité des racines (Kramer, 1983). Ainsi, au moins 2 éléments distincts, tension de l'eau plus forte et oxygénation réduite, pourraient contribuer à diminuer les possibilités d'alimentation hydrique des arbres sur sol fin en hiver. Nous menons actuellement une étude sur le terrain pour estimer dans quelle mesure ces éléments interviennent pour expliquer les différences de teneur en eau constatées. Quelques résultats concernant, dans un premier temps, la tension de l'eau dans le sol semblent confirmer une telle possibilité. Les mesures réalisées sur les pêchers en conteneur ont donné des indications dans le même sens d'autant que, dans cette expérience, le substrat était le même; seul le facteur eau a été modifié.

On peut rapprocher ces situations de celle décrite par Matthee et Daines en 1968. Elle concernait des feuilles de pêchers en végétation exposés à une hygrométrie saturante. Dans ces conditions, une infiltration hydrique, très semblable (même si elle est d'origine différente) à celle que nous étudions, se développe. Dans le cas rapporté par ces auteurs, le phénomène a 
été accru chez des arbres cultivés sur sable, comparé à celui obtenu sur des arbres cultivés sur un limon fin, où une infection bactérienne à Xanthomonas, provoquée sur les feuilles, a été plus grave.

Ainsi, dans le cas du dépérissement bactérien du pêcher, on a montré qu'une modification hivernale des conditions de sol a pu modifier la teneur en eau hivernale de jeunes pêchers et modifier dans le même sens leur sensibilité à l'infection. En parallèle, on a constaté que la culture sur sols grossiers qui favorise fortement la maladie semble augmenter systématiquement la teneur en eau hivernale des pêchers qu'ils portent. On avait auparavant obtenu des variations de sensibilité de rameaux de pêchers dormants excisés en modifiant leur teneur en eau (ct introduction). On peut donc penser qu'un des éléments de la prédisposition des pêchers à la bactériose réside en la modification de leur teneur en eau hivernale qui pourrait découler, entre autres, d'une action physique des sols de verger.

Dans une perspective plus globable, le phénomène s'intègre dans un système où, en plus des facteurs froid et nutrition déjà cités, interviennent aussi d'autres facteurs climatiques ou culturaux (brouillard, vigueur, taille hivernale...) susceptibles de faire varier la teneur en eau hivernale des pêchers (Vigouroux, article en préparation). La diversité des facteurs impliqués rendrait alors compte au moins pour une part de la grande irrégularité des manifestations de ce type de maladie (Paulin, 1994), qui, compte tenu de ces divers impératifs, ne pourrait revêtir un caractère grave qu'à la suite de la conjonction d'un ensemble complexe de circonstances.

On doit, par ailleurs, relever que l'ensemble des bactérioses hivernales à Pseudomonas des arbres fruitiers à noyau, outre le caractère hivernal des infections et l'agent bactérien responsable (toujours un Pseudomonas syringae), présente beaucoup de caractères communs ou très semblables (Vigouroux, 1984) : symptômes, cycle du parasite, effets du sol (cf introduction). Nous avons aussi constaté que tous ces arbres sont affectés par le phénomène d'infiltration hydrique après gel et la diffusion passive de germes qui lui est associée (Vigouroux, 1989 ; et résultats non publiés). II est donc envisageable que le mode d'action des sols que nous avons présenté puisse concerner tout cet ensemble de maladies bactériennes.

\section{REMERCIEMENTS}

Ce travail est soutenu par un financement de la région Languedoc-Roussillon. Nous avons apprécié l'aide technique de Mme V Chalvon et M T Girard, ainsi que la collaboration des propriétaires des vergers, supports de ce travail.

La souche de Pseudomonas $\mathrm{S} 32$ nous a été aimablement fournie par nos collègues $\mathrm{J}$ Luisetti et $\mathrm{J}$ Gaignard du laboratoire de phytobactériologie d'Angers.

\section{RÉFÉRENCES}

Bornand M (1968) Étude pédologique dans la vallée du Rhône. Plaine de Valence-Romans. Rapport du service d'étude des sols. INRA, Montpellier, $96 \mathrm{p}$

Bornand M (1978) Altération des matériaux fluvio-glaciaires, genèse et évolution des sols sur terrasses quaternaires dans la moyenne vallée du Rhône. Thèse doctorat d'état, Univ Montpellier, $329 \mathrm{p}$

Burke MG, Gusta LV, Quamme MA, Weiser CJ, Li PH (1976) Freezing and injury in plants. Annu Rev Plant Physiol 27, 507-528

Chrétien J, Tessier D (1988) Influence du squelette sur les propriétés physiques des sols, hydratation, glonflement, aération. AFES Sci Sol 4, 255-267

Davis JR, English H (1969) Factors related to the development of bacterial canker in peach. Phytopathology 59, 588-595

Fitter AH, Hay RK (1987) Environmental Physiology of Plants. Acad Press, London, $2^{\mathrm{e}}$ ed, $423 \mathrm{p}$

Gaignard JL, Luisetti J (1993) Pseudomonas syringae, bactérie épiphyte, glaçogène et pathogène. agronomie 13, 333-370

Geneves L (1955) Recherches sur les effets cytologiques du froid. Rev Cytol Bio Veg XVI (1-2), 1-207

Gras R (1962) Quelques observations sur les relations entre les propriétés physiques du sol et la croissance du pêcher dans la vallée du Rhône entre Vienne et Valence. Ann Agron 13(2), 141-174

Klément Z, Roznyay DS, Arsenijevic M (1974) 11. Relationship of winter frost and the bacterial canker and dieback of apricots. Acta Phytopathol Acad Sci Hung 9, 35-45

Klément Z, Rozsnyay DS, Balo E, Panczel M, Prileszky Gy (1984) The effect of cold on development of bacterial canker in apricot trees. Physiol Plant Pathol 24, 237-246

Kramer PJ (1983) Water Relations of Plants. Acad Press, New York, $385 \mathrm{p}$

Levitt J (1956) The Hardiness of Plants, Acad Press, New York, $258 \mathrm{p}$

Lévy L, Toutain F (1979) Aération et phénomènes d'oxydo-réduction dans le sol. In: Pédologie, 2 : constituants et propriétés du sol (P Duchauffour, B Souchier, eds), Masson, Paris, $437 p$ 
Luisetti J, Gaignard JL (1985) Les bactéries glaçogènes et les gelées de printemps. In: $C C 5^{e} \mathrm{col}$ Recherches Fruitières Bordeaux, 13-14 novembre, Éd CTIFL, 33140 Pont-de-la-Maye, France, 67-73

Maki ML, Galyan EL, Chang Chien MM, Caldwell DR (1974) Ice nucleation induced by Pseudomonas syringae. Appl Microbiol 28, 456-459

Matthee FN, Daines RH (1968) The effect of soil types and substrate aeration on stomatal activity, water diffusion, pressure deficit, water congestion and bacterial infection of peach and pepper foliage. Phytopathology 58, 1298-1301

Marty JR (1969) Contribution à l'étude des boulbènes. Thèse doctorat d'univ, Paris. $135 p$

Olien CR (1967) Freezing stresses and survival of plants. Annu Rev Plant Physiol 18, 387-408

Panagopoulos CG, Crosse JE (1964) Frost injury as a predisposing factor in blossom blight of pear caused by Pseudomonas syringae Van hall. Nature 202, 1352

Paulin JP (1994) Chemical and biological control of bacterial diseases: a need. In : Proc 8th Inter Conf Plant Path Bact, Versailles 9-12 June 1992. INRA

Ridé M (1963) Connaissances actuelles sur le chancre bactérien du peuplier dû à Aplanobacterium populi. Publ FAO, Int Poplar Comm Res Dis Group, 9 p

Sakai A, Yoshida S (1968) The role of sugar and related compounds in variations of freezing resistance. Cryobiology 5, 160-174

Vigouroux A (1974) Obtention de symptômes de bactériose du pêcher sur rameaux de pêcher détachés. Effets du froid. Ann Phytopathol 6, 95-98

Vigouroux A (1979) Incidence des basses températures sur la sensibilité du pêcher au dépérissement bactérien. Ann Phytopathol 11, 231-239

Vigouroux A (1984) Verticilliose et bactériose, deux importants facteurs de dépérissement de l'abricotier. Arbor fruitière 365, 31-35

Vigouroux A (1989) Ingress and spread of Pseudomonas in stems of Peach and Apricot promoted by frost-related water soaking of tissues. Plant Dis 73, 854-855
Vigouroux A (1991a) Mechanism of cold-induced peach stem infection by Pseudomonas s pv persicae: water congestion in tissues. J Phytopathol 132, 139-145

Vigouroux A (1991b) Influence du froid et de la teneur en eau sur la prédisposition des tiges de pêcher aux infections à certains Pseudomonas. In : "L'arbre" (C Edelin, ed) Naturalia Monspeliensia ${ }^{\circ}$ hs

Vigouroux A, Huguet $C$ (1980) Prédisposition du pêcher au dépérissement bactérien sur sol d'arène et de diluvium. Ann Phytopathol 12 (4) 312

Vigouroux A, Bussi C (1989) Importance of water consumption on calcium content and protection of peaches predisposed to bacterial dieback by growing in acid soils. Acta Hortic 254, 291-296

Vigouroux A, Bussi C (1994) Pseudomonas canker of stone-fruit trees: a probable predisposing effect of coarse soils by increase of the twig water content in winter. Proc 8th Inter Conf Plant Path Bact, Versailles 9-12 June 1992, INRA éd

Vigouroux A, Berger JF, Bussi C (1987) La sensibilité du pêcher au dépérissement bactérien en France : incidence de certaines caractéristiques du sol et de l'irrigation. Relations avec la nutrition. agronomie 7, 483-495

Weaver DJ (1978) Interaction of Pseudomonas syringae and freezing in bacterial canker on excised peach twigs. Phytopathology 68, 1460-1463

Weaver DJ, Wehunt EJ (1975) Effect of soil pH on susceptibility of peach to Pseudomonas syringae. Phytopathology 65, 984-989

Weaver DJ, Gonzalez CF, English H (1981) Ice nucleation by Pseudomonas syringae associated with canker production in peach. Phytopathology 71,109 (abstr)

Wiegand KM (1906) Some studies regarding the biology of buds and twigs in winter. Bot Gaz 41, 373424

Yong RN, Warkentin BP (1975) Water retention in soil. In : Soil Properties and Behaviour. Elsevier ed, $449 p$

Young JM (1974) Effect of water on bacterial multiplication in tissues. NZJ Agric Res 17, 115-119 